\title{
The dilemma of future family planning and the role of female fertility tests
}

\author{
Andrew Horne, Pam Warner
}

\section{Introduction}

There has been a huge amount of media interest in the development of new home fertility tests for couples trying to conceive. The female fertility tests are marketed as devices that measure the quantity of healthy eggs women have left (ovarian reserve). Ovarian reserve is measured by determining the level of follicle-stimulating hormone (FSH) in the urine. High levels of FSH suggest that the ovaries are not responding to the hormone and that the woman's ability to conceive may be decreased. The tests show whether the FSH level is above healthy cut-off levels. One test is being marketed particularly to help women decide how long they can delay trying for a baby. In addition to FSH, it also measures inhibin B and anti-Müllerian hormone levels, which decline as the menopause approaches.

\section{Clinical scenario}

A 29-year-old woman, para 0+0, attends your practice for advice. She has recently purchased one of the new home fertility test kits. Her results suggest that her ovarian reserve is low for her age. She has no current partner.

\section{The panel}

Four health professionals and a lay person (detailed in Box

1) were invited to discuss what they would have done.

\begin{tabular}{|c|}
\hline $\begin{array}{l}\text { Box } 1 \text { Invited discussants for the clinical scenario } \\
\text { - Ethicist } \\
\text { - Medical sociologist } \\
\text { - Lay persogist } \\
\text { - General practitioner }\end{array}$ \\
\hline
\end{tabular}

\section{Ethicist}

This is a kind of case that we shall see a lot more of. We can find out about a woman's ovarian reserve, but at the moment we have limited power to assure a healthy pregnancy at a future date. Egg storage and ovarian tissue storage are uncertain options, though no doubt the capacity to produce a pregnancy from these will improve.

As a society, we are starting to think more globally about ethical issues. We are aware of overpopulation and the hazards of selfishness for the environment, as well as the millions of unwanted children in the world. All this, I believe, must inform a serious public debate about the 'pros and cons' of various kinds of medical technology. However, this is a policy perspective, and I would not want to confuse this with the response to the 29-year-old woman in this scenario.

\section{J Fam Plann Reprod Health Care 2006; 32(3): 195-196}

Simpson Centre for Reproductive Health, University of Edinburgh, Royal Infirmary of Edinburgh, Edinburgh, UK Andrew Horne, PhD, MRCOG, Clinical Lecturer

\section{University of Edinburgh Medical School, Public Health Sciences, Edinburgh, UK \\ Pam Warner, PhD, Statistician}

Correspondence to: Dr Andrew Horne, Simpson Centre for Reproductive Health, Royal Infirmary of Edinburgh, 51 Little France Crescent, Edinburgh, EH16 4SA, UK. E-mail: awhorne@hotmail.com
This woman has sought knowledge about her ovarian reserve. In order to treat her with the respect that is due to every individual, we must do our best to make sure (a) that the knowledge is accurate and (b) that she understands it correctly. The woman should be offered more tests and an assessment of the reliability of their results. She should be told not merely that her ovarian reserve is 'low for her age', but what age it is roughly equivalent to. Is this reserve found on average in 33-year-old women? In this case, her chances of conceiving during the next 5 years or so would be good, though she may want to repeat tests in a year in order to confirm this. I would respect the woman's desire for knowledge, and try to quell her anxiety.

\section{Medical sociologist}

The meeting of biology and culture is probably nowhere more pronounced than in the field of human reproduction. Women clearly have the right to control their own bodies and the provision of relevant health information may support that. However, we also know that women's bodies, particularly where reproduction is concerned, are also the subject of control and surveillance. The media interest in women's 'delayed childbearing' and childlessness (voluntary or involuntary) has both an individual and societal focus. Falling birth rates are considered a social issue as many Western countries struggle to achieve replacement level, yet decisions when or whether to have children are deeply personal and not always planned. The commercial test may evoke anxiety, suggest a fertility problem where none exists, and lead to action that may not be in the best interests of the woman.

As a sociologist, I would hope this doctor remains mindful of this wider framing of what might otherwise be portrayed as a biological issue, listen to the young woman and provide advice about how to maintain reproductive health. The doctor should warn about the inaccuracies and limits of the home fertility test and stress that this is just one measure of fertility. There are many different family forms within which children are born and raised and the doctor should be supportive of this. However, the results of one test should not precipitate a hasty decision incompatible with the woman's own circumstances and inclinations.

\section{Gynaecologist}

The average age of the menopause in Western societies is around 52 years, preceded by a progressive decline in fertility from the mid-20s onwards. Despite this, most women remain capable of conceiving a pregnancy into their early 40s, and premature menopause under the age of 40 is rare. It would be relevant to ask this woman if she has a family history of early menopause and whether she has developed any symptoms suggestive of the onset of menopause.

There are two separate components to this decline in fertility. One is the number of follicles in the ovary capable of maturing to produce eggs (oocytes), known as the ovarian reserve. The other is the quality of the oocytes. Both components decline with age but the blood tests of ovarian reserve assess only the quantity and not the quality of the oocytes. During any one menstrual cycle, young 


\section{CLINICAL CONUNDRUM}

women at the peak of fertility have a large number of immature ovarian follicles capable of developing and releasing eggs but only one of these will achieve ovulation. The others undergo atresia. This monthly wastage of potential oocytes occurs even in women taking contraceptives such as the pill. As women get older the number of available follicles declines, as reflected in the results of these blood tests. However, as long as there is at least one follicle capable of releasing a healthy oocyte each month, conception remains possible.

Blood tests of ovarian reserve have been assessed in women attending fertility clinics and undergoing treatment such as in vitro fertilisation (IVF). The results are quite good at predicting the numbers of oocytes that can be obtained at IVF but rather poor at predicting whether treatment will result in a pregnancy. The tests have not been properly and prospectively evaluated in a general population of women and their commercial availability is arguably premature.

What advice should we give this woman? Despite extensive media coverage there are currently limited options for preserving fertility. Egg storage is available at a cost but resultant pregnancy rates are very low. Ovarian tissue storage is offered to some women at risk of losing their fertility from cancer treatment but techniques are experimental and involve invasive surgical procedures. Single parenthood using donor sperm for artificial insemination or IVF with embryo storage has limited availability in the UK because of a donor shortage due to loss of anonymity. Does she want to be a single parent? Unless she has actually developed any symptoms of an imminent menopause, it would be difficult to justify any of these options given the lack of evidence for the predictive value of the test. Even if its predictions are correct, they reflect only a decline in oocyte numbers, and she should be reassured that her age remains a favourable factor in relation to the quality of her eggs and her prospects for future fertility.

\section{Lay person (29-year-old woman, para 0+0)}

First, the fact that the woman has taken a fertility test at all suggests that she is at some level enquiring into having a child (even if she has not openly admitted this to herself). The results seem to provide weight to the 'pro-baby' choice but this is not enough for what will be a significant, lifechanging commitment. She will now be looking not just for information but, more importantly, for indicators, reinforcement and courage to support her decision. Arguably, she already knows at a deep level what she wants to do, but the role of the expert is crucial in helping her get to this inner instinct. As any external adviser will not know which way this instinct is bearing (initially), a balanced set of information will be vital. Key questions to be answered would include the following: how reliable the test is, how quickly the ovarian reserves deplete, what is the chance of pregnancy with different hormone levels, how quickly a new couple trying for a baby are likely to get pregnant, and what the options are for an artificially created conception including success rate, availability, cost, any health contraindications, and so on. This would cover the practical needs from the patient's point of view and should provide a useful basis for a decision on how and whether to have a child as prompted by the new health tool.

However, what is not covered in this approach so far is any discussion of what it means to turn the traditional order of procreation (love + partnership + baby) on its head, and seeking the child before the relationship that would create it. My personal view is that if the test is reliable it could be treated as a helpful additional 'prompt' to taking the huge, uncomfortable but ultimately rewarding risk of both committing to a partner and then being open to having a child. But what doesn't work (for me) is to try to 'acquire' a child because nature appears to have given you a time limit (or a shorter than average one). I feel that a child, or even our ability to have a child, is a gift - not something that can or should be manufactured. Perhaps women are going to have to accept that not everything can or should be grabbed like a box of soap powder off the shelf, and that life works better when we accept what we are given. In an overpopulated, over-consuming world perhaps we do not all have the right to have whatever we want, particularly when that is another human life.

\section{General practitioner}

I would initially like to establish why this woman decided to buy and carry out this relatively costly test. She may simply have been curious, perhaps her cycle is a little irregular, her periods are infrequent and she was seeking reassurance that she was fertile and indeed had many fertile years ahead. It may be that she has a friend or relative who had a premature menopause and she was concerned she may be in a similar position.

Next, in a more practical vein, I would wish to know if she had carried out the test as instructed (i.e. on the third day of her cycle) and that she had interpreted the result properly. I understand that the intensity of the colour of the result line indicates the amount of FSH being excreted and wonder if this might be open to misinterpretation.

I would then wish to establish what she had hoped I might do to help her and what her immediate and long-term concerns are. It may be that she wants me to carry out a test to confirm or refute the findings of her home test. She may be hoping I might be able to give her some kind of timescale within which she should become pregnant. She may be thinking as far ahead as wishing referral to a specialist clinic to have her eggs frozen.

I would wish to know what her menstrual cycle was like and also ask about perimenopausal symptoms to ensure this was not an immediate cause for concern. Then, depending on what her hopes and expectations for the consultation were, I would try and address these. I understand that the test detects not only FSH but also a couple of other hormones, and that such tests are not currently available to us in general practice. In terms of repeat testing, all I would be able to do is check FSH/luteinising hormone levels, which may not be helpful.

Frequently in practice young women who have an irregular cycle but who are not trying to conceive ask me: "I am worried that means I won't be able to get pregnant. Can I be referred to the hospital to be checked out?" In such circumstances I advise these women that I wouldn't investigate this unless they were failing to conceive. This case is different, I feel. She has done this test and can now almost hear her biological clock ticking.

I would advise her that I do not think anyone would be able to offer her a timescale for conception if this was what she was looking for, but that I would be happy to write to the local infertility clinic asking their advice on her case. I am aware of a local clinic that offers the freezing of eggs but do not know who is eligible for this service, if indeed this option were to be appropriate in the present case. I would advise her that if she did not want to delay and wait for feedback from the infertility clinic then she could always be referred privately there.

\section{Acknowledgements}

The authors would like to thank the panel members for their input. A listing of the individual panel members who have contributed to the Clinical Conundrum section of the Journal is published annually. 